\title{
Chitosan Interferon- $\gamma$ Nanogene Therapy for Lung Disease: Modulation of T-Cell and Dendritic Cell Immune Responses
}

Xiaoyuan Kong, MD, Gary R. Hellermann, PhD, Weidong Zhang, PhD, Prasanna Jena, PhD, Mukesh Kumar, PhD, Aruna Behera, PhD, Sumita Behera, MSc, Richard Lockey, PhD, and Shyam S. Mohapatra, PhD

The use of chitosan nanoparticles as carriers for expression plasmids represents a major improvement in gene expression technology. We demonstrated previously that treatment with chitosan interferon- $\gamma$ (IFN- $\gamma$ ) plasmid deoxyribonucleic acid (DNA) nanoparticles (chitosan interferon- $\gamma$ nanogene [CIN]) led to in situ production of IFN- $\gamma$ and a reduction in inflammation and airway reactivity in mice, but the mechanism underlying the immunomodulatory effects of CIN remains unclear. In this report, the effect of CIN treatment on the immune responses of $\mathrm{CDB}^{+} \mathrm{T}$ cells and dendritic cells was examined in a BALB/c mouse model of ovalbumin (OVA)-induced allergic asthma. OT1 mice (OVA-T cell receptor [TCR] transgenic) were also used to test the effects of CIN on OVAspecific $\mathrm{CD}^{+} \mathrm{T}$ cells. CIN treatment caused a reduction in IFN- $\gamma$ production in a subpopulation of OVA-specific CD8 ${ }^{+} \mathrm{T}$ cells cultured in vitro in the presence of OVA. CIN also reduced apoptosis of the CD8 ${ }^{+} \mathrm{T}$ cells. Examination of dendritic cells from lung and lymph nodes indicated that CIN treatment decreased their antigen-presenting activity, as evident from the reduction in CD80 and CD86

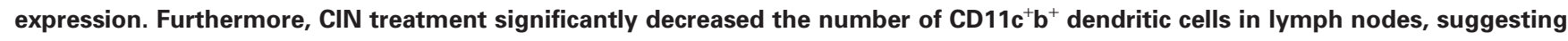
that endogenous IFN- $\gamma$ expression may immunomodulate dendritic cell migration and activation. CIN therapy results in a reduction in proinflammatory $\mathrm{CDB}^{+} \mathrm{T}$ cells and decreases the number and antigen-presenting activity of dendritic cells.

Key words: allergy, asthma, interferon

$T^{\text {he past decade has seen tremendous progress in gene }}$ 1 expression technology. Several investigators, including us, have used viral vectors for transient gene expression with some success. The replication-deficient episomal adenovirus has been the workhorse for gene therapy, but

Xiaoyuan Kong, Gary R. Hellermann, Weidong Zhang, Prasanna Jena, Mukesh Kumar, Aruna Behera, Sumita Behera, and Shyam Mohapatra: Division of Allergy and Immunology, Culverhouse Airway Disease Research and Nanomedicine Center, University of South Florida College of Medicine, Tampa, FL; and Richard Lockey: James A. Haley VA Medical Center, Tampa, FL.

These studies were supported by grant 5RO1 HL 071101-02 and VA Merit Review and Career Scientist Award to S.S.M and the Florida Biomedical Research Foundation Bankhead-Coley Award and Mabel and Ellsworth Simmons Professorship to S.S.M., and by the Joy McCann Culverhouse endowment to the University of South Florida Division of Allergy and Immunology.

Correspondence to: Shyam S. Mohapatra, $\mathrm{PhD}$, Division of Allergy and Immunology, Joy McCann Culverhouse Airway Disease Research Center, University of South Florida College of Medicine, Box MDC-19, 12901 Bruce B. Downs Blvd, Tampa, FL 33612; e-mail: smohapat@ health.usf.edu.

DOI $10.2310 / 7480.2008 .00006$ its toxicity and immunogenicity limit its clinical use. ${ }^{1-5}$ Consequently, we have developed and tested a nonviral platform for gene expression using plasmid deoxyribonucleic acids (pDNAs) that offers ease of preparation and use, in vivo stability, heat resistance, and the capacity for large DNA sequences. The plasmids do not integrate into mammalian genomes or replicate, yet they can persist in host cells and express the cloned gene for several months. The drawback of pDNA is its relatively low transfection efficiency under physiological conditions, especially in non-dividing or slowly dividing cells, such as epithelial cells. Some improvement in the transfection efficiency of pDNA has been made using liposomes or receptor targeting, ${ }^{6,7}$ but the approaches remain largely empirical.

One important development in gene transfer was the discovery that chitosan (a biocompatible cationic polysaccharide derived from crustacean shell chitin) in the form of nanoparticles (100-200 $\mathrm{nm}$ ) could be used to deliver plasmids. ${ }^{8-12}$ Chitosan has beneficial immunostimulatory, ${ }^{13}$ anticoagulant, ${ }^{14}$ wound-healing, ${ }^{15}$ and antimicrobial properties. ${ }^{16}$ It is nontoxic in humans, nonhemolytic, weakly immunogenic, and slowly biodegradable and has been widely used in controlled drug delivery. ${ }^{9,17-21}$ 
It also has mucoadhesive properties that increase transcellular and paracellular transport across the mucosal epithelium, ${ }^{22}$ which should facilitate gene delivery to mucosa- and bronchus-associated lymphoid tissue. Chitosan, therefore, appears to possess all of the attributes of an ideal gene delivery agent for effective nonviral gene expression therapy.

Since its discovery, interferon- $\gamma$ (IFN- $\gamma$ ) has been extensively studied for its immunomodulatory and antiviral activity. Mice lacking the IFN- $\gamma$ receptor exhibit a T-helper (Th)2-like cytokine profile, implying that IFN- $\gamma$ may be a key cytokine in asthma. ${ }^{23}$ IFN- $\gamma$ provides the stimulatory signal for interleukin (IL)-12, ${ }^{23,24}$ which is a strong inducer of the Th1 response. IL-12 inhibits Th2 cells by downregulating the production of IL-4 and IL-5. The IFN- $\gamma$ inducing factor IL-18 also shifts the immune response from a Th2 to a Th1 state. ${ }^{25,26}$ Local administration of aerosolized IFN- $\gamma$ prevented antigen-induced eosinophil recruitment in guinea pig trachea. ${ }^{27}$ IFN- $\alpha$, IFN- $\beta$, and $-\gamma$ all inhibit leukotriene $\mathrm{C}_{4}$ production in murine macrophages, ${ }^{28}$ but IFN- $\gamma$ treatment of guinea pigs induced the release of prostanoids and nitric oxide, which modify airway smooth muscle responses through effects on airway epithelium. ${ }^{29}$

Patients with allergic asthma exhibit lower than normal production of IFN- $\gamma$ and IFN- $\gamma$-dependent IL-12 in whole blood cultures after stimulation with a mitogen. ${ }^{30}$ Because of the reciprocal regulation of $\mathrm{T}$ helper cells, it was anticipated that increasing IFN- $\gamma$ levels via chitosan pIFN$\gamma$ nanoparticles (chitosan interferon- $\gamma$ nanogene $[\mathrm{CIN}]$ ) would promote a Th1 response by blocking Th2 cytokine production. IFN $-\gamma$ upregulates the IL-13R $\alpha 2$ decoy receptor, leading to diminished IL-13 signaling $^{31}$ and reduced goblet cell hyperplasia and eosinophilia. ${ }^{32}$ IFN- $\gamma$ significantly inhibits release of leukotrienes from peripheral blood leukocytes (PBLs) of allergic individuals after wasp venom immunotherapy ${ }^{33}$ and decreases the production of sulfidoleukotrienes by human PBLs. ${ }^{34}$ IFN- $\gamma$ also downregulates transforming growth factor $\beta$ and procollagen I and III and decreases fibrosis in a mouse model of bleomycin-induced lung injury. ${ }^{35}$

Exogenously supplied cytokines, such as IFN- $\gamma$, IL-12, and IL-18, have a short half-life in vivo, and systemic administration at moderate to high doses can cause substantial adverse effects. ${ }^{36,37}$ To overcome these limitations to their therapeutic use, several investigators have used expression vectors containing the cloned cytokine complementary deoxyribonucleic acid (cDNA) under the regulation of a constitutive promoter as a means of boosting in vivo production of specific cytokines. IFN- $\gamma$ and IL-12 have proven effective as prophylactics and adjuncts in therapy against diverse human diseases. ${ }^{38,39}$ IL-12 gene transfer and expression in the mouse airway abrogated airway eosinophilia and immunoglobulin E (IgE) synthesis, ${ }^{40}$ and benefits from IFN- $\gamma$, IL-12, and IL-18 gene therapy have been documented in other animal models. ${ }^{41-43}$ However, the methods used to transduce plasmids in mice are not directly applicable to humans because of the use of lipofectamine, which is toxic to humans.

Oromucosal therapy with recombinant IFN reduced the severity of viral infection, ${ }^{44}$ but intranasal administration of IFN- $\gamma$ pDNA has not been tested. Previous studies from our laboratory demonstrated that intranasal administration of IFN- $\gamma$ and IL-12 plasmids inhibited the induction of IL-5 messenger ribonucleic acid (mRNA), afforded protection against viral infections, and significantly decreased airway inflammation and airway hyperresponsiveness. In this article, we investigated the mechanism of $\mathrm{CIN}$-mediated immunomodulation using the mouse OVA-allergic asthma model. IFN- $\gamma$ treatment reduced cytokine production by a population of OVA-specific proinflammatory $\mathrm{CD} 8^{+} \mathrm{T}$ lymphocytes in the lung and led to decreased activation of dendritic cells.

\section{Materials and Methods}

\section{Animals}

All mice were purchased from Jackson Laboratory (Bar Harbor, ME). BALB/c mice have a predominantly Th2type response to allergens, and these were used for most of the experiments. The transgenic C57BL/6$\mathrm{TG}(\mathrm{TcraTcrb}) 1100 \mathrm{mjb} / \mathrm{j}$ mice have $\mathrm{CD}^{+} \mathrm{T}$ cells specific for ovalbumin (OVA) amino acids 257 to 264, and these animals were used in experiments to examine CIN effects on $\mathrm{CD}^{+}$cells. Female 6- to 8-week-old mice were maintained in pathogen-free conditions at the University of South Florida College of Medicine vivarium. All procedures were reviewed and approved by the Committee on Animal Research at the University of South Florida College of Medicine and VA Hospital. A minimum of four mice were used in each test group, and experiments were repeated twice.

\section{Preparation of Chitosan IFN- $\gamma$ pDNA Nanoparticles and Green Fluorescent Protein Test of Expression}

Mouse IFN $-\gamma$ cDNA was cloned in the mammalian expression vector pVAX (Invitrogen, San Diego, CA), and complexed with chitosan, as described previously. ${ }^{45}$ Briefly, plasmids in $25 \mathrm{mM} \mathrm{Na}_{2} \mathrm{SO}_{4}$ and chitosan (Vanson, 
Redmond, WA) dissolved in $25 \mathrm{mM} \mathrm{Na}$ acetate, $\mathrm{pH}$ 5.4, to a final concentration of $0.02 \%$ were separately heated for 10 minutes at $55^{\circ} \mathrm{C}$. After heating, the chitosan and DNA were mixed, vortexed vigorously for 20 to 30 seconds, and stored at room temperature until use. This treatment results in nanoparticles of 200 to $300 \mathrm{~nm}$ diameter as measured in transmission electron micrographs and by dynamic light scattering. The effectiveness of intranasal chitosan nanoparticle-mediated gene transfer in mice was tested with a vector that expresses green fluorescent protein (plasmid-encoding green fluorescent protein [pEGFP]). Chitosan nanoparticles complexed with pEGFP were prepared as above, and an amount containing $10 \mu \mathrm{g}$ of pEGFP was administered intranasally to mice. After 1, 3, and 7 days, the mice were euthanized and the lungs were removed and fixed in buffered formalin. Whole lungs were embedded in paraffin, sectioned, and examined for GFP by fluorescent microscopy, and an approximate percentage of GFP-positive cells was estimated.

\section{OVA Sensitization, CIN Treatment, and Preparation of Lung Sections}

Mice were allergen-sensitized by intraperitoneal injection of $50 \mu \mathrm{g}$ of OVA adsorbed to $2 \mathrm{mg}$ of alum (Imject, Pierce, Rockford, IL) followed by an intranasal challenge with 50 $\mu \mathrm{g}$ of OVA after 1 or 2 weeks. To test the effects of boosting IFN- $\gamma$ production on allergen sensitization, mice were given an intranasal treatment of $10 \mu \mathrm{g}$ of $\mathrm{CIN}$, chitosan with vector, or chitosan alone prior to intraperitoneal injection of OVA-alum. This constitutes the prophylactic CIN regimen. Other groups of mice were given CIN treatments after OVA sensitization as a therapeutic regimen. In both prophylactic and therapeutic treatments, the mice were given a final challenge with OVA and then euthanized, and the lungs from mice from each group were perfused in situ with phosphate-buffered saline (PBS), removed, and fixed in $4 \%$ buffered formalin. Lungs from some mice in each group were left unfixed and homogenized for lymphocyte isolation (see below). Whole formalin-fixed lungs were paraffin-embedded, and 15micron sections were made. Two sections from each mouse were dewaxed, rehydrated, and labelled with either anti-IL-12Rb2-fluorescein isothiocyanate (FITC) (Th1) or anti-T1/ST2L-rhodamine (Th2). Viewers examining the stained sections were blinded as to the mouse group from which the slides were taken. The stained sections were examined with a Nikon TE300 fluorescence microscope, and representative areas were photographed.

\section{Isolation of Lung Cells and Flow Cytometry of IFN- $\gamma$ - Producing, OVA-Specific $\mathrm{CD8}^{+} \mathrm{T}$ Lymphocytes}

C57BL/6 mice transgenic for the OVA-specific $\mathrm{T}$ cell receptor (TCR) (amino acids 257-264) and C57BL/6 wildtype controls were given intranasal CIN treatment $(10 \mu \mathrm{g})$, followed by intraperitoneal sensitization with OVA-alum on day 1 . On day 10 , the mice were again treated with CIN and then challenged intranasally with OVA. Twenty-four hours after the challenge, the mice were euthanized and the lungs were perfused with PBS and removed. Lungs were weighed, minced, and homogenized with Teflon homogenizers. The homogenates were digested with collagenase $(50 \mathrm{U} / \mathrm{mL})$ in the presence of DNase I $(200 \mu \mathrm{g} / \mathrm{mL})$ and passed through a 40-micron cell strainer to prepare single-cell suspensions. This is a standard method of cell preparation and does not result in selective loss or enrichment of lymphocytes or dendritic cells. Viabilities by trypan blue dye exclusion were $>80 \%$. The cells were cultured for 20 hours with $50 \mu \mathrm{g} / \mathrm{mL}$ OVA. Cultures were treated with $5 \mu \mathrm{g} / \mathrm{mL}$ brefeldin A for 4 hours prior to harvesting to block secretion of intracellular cytokines. Class I restricted $\mathrm{CD}^{+} \mathrm{T}$ cells were surfacestained with OVA tetramer consisting of the OVA peptide Ser-Ileu-Ileu-Asn-Phe-Glu-Lys-Leu, bound to four major histocompatibility complex (MHC)-I molecules conjugated with a fluorescent phycoerythrin tag (Beckman/Coulter Immunomics, Fullerton, CA) and intracellularly stained with FITC-conjugated anti-IFN- $\gamma$. Cells were counted by flow cytometry (FACScan, BD Biosciences, San Jose, CA) with side-scatter/forward-scatter set for lymphocytes and gating for $\mathrm{CD}^{+}$tetramer. Unstained cells were run as a control, and non-viable cells were distinguished by staining with 7-amino-actinomycin D. Tetramers use a mutated form of MHC-I with low binding affinity to CD8 on nonOVA-recognizing $\mathrm{T}$ cells, so there is no need to run a tetramer control.

\section{Apoptosis of OVA-Specific CD8 ${ }^{+}$T Lymphocytes from Lung}

OVA-sensitized OT-1 mice were given therapeutic CIN treatment and 24 hours later were challenged intranasally with OVA. Mice were euthanized 18 hours after OVA challenge. Lungs were removed, homogenized, digested with collagenase and DNase, and passed through a cell strainer to produce single-cell suspensions. The cells were pelleted by centrifugation at $500 \mathrm{~g}$ for 5 minutes at $4{ }^{\circ} \mathrm{C}$, and then red blood cells were lysed by resuspending the cell pellets twice in ice-cold lysis buffer $\left(0.156 \mathrm{M} \mathrm{NH}_{4} \mathrm{Cl}\right.$, $10 \mathrm{mM} \mathrm{KHCO} 3,0.1 \mathrm{mM}$ ethylenediaminetetraacetic acid, 
pH 7.3). Cells were resuspended in Roswell Park Medium I (RPMI) 1640 , plated, and incubated at $37^{\circ} \mathrm{C}$ for 18 hours. Non-adherent cells consisting primarily of lymphocytes were removed by pipetting off the medium. T lymphocytes were isolated by mouse T-cell enrichment columns ( R \& D Systems, Minneapolis, MN) and analyzed for apoptosis using the TUNEL (terminal deoxynucleotidyl nick endlabeling) assay (Promega, Madison, WI). The OVAspecific $\mathrm{CD}^{+}$T-cell population was labelled with phycoerythrin-tagged tetramer for OVA peptide conjugated with MHC-I (Beckman/Coulter Immunomics). Unstained cells were used as a control. The number of apoptotic OVA-specific $\mathrm{CD}^{+} \mathrm{T}$ cells was determined by flow cytometry (FACScan, BD Biosciences) and expressed as a percentage of the total number of OVA-specific $\mathrm{CD} 8^{+}$cells.

\section{Analysis of the Dendritic Cell Population in Lung Parenchyma}

OVA-allergic BALB/c mice were treated therapeutically with CIN and then challenged with OVA and euthanized 18 hours later. Lungs were removed, and single-cell suspensions were prepared and plated as described above. In this case, however, mononuclear cells were isolated from the cells that had adhered to the dishes after removal of the lymphocytes. These were further purified using magnetic beads coated with anti-CD11c (Miltenyi Biotec, Auburn, CA) with cell recoveries in the range of 3 to 5 million per gram of lung tissue. The $\mathrm{CD} 11 \mathrm{c}^{+}$cells were then seeded into 12 -well plates at $5 \times 10^{5}$ cells per well and cultured for 48 hours in the presence of $10 \mu \mathrm{g}$ of chitosanpVAX (vector control) or CIN. Cells were scraped from the wells, resuspended in PBS $+3 \%$ fetal calf serum (FCS), stained with phycoerythrin meaning (PE)-antiCD11c and individually with FITC-anti-I-Ad, -CD40, and -CD80. $\mathrm{CD}_{11 \mathrm{c}^{+}}$cells expressing each of the dendritic cell activation markers were counted by flow cytometry (FACScan, BD Biosciences) with appropriate controls.

In another set of experiments, lung mononuclear cells were isolated as described above but were not cultured. The cells were stained with FITC-anti-CD11c and rhodamineanti-CD11b and counted by flow cytometry (FACScan, BD Biosciences). The numbers of CD1 $1 c^{+} b^{+}$cells were expressed as a percentage of the total $\mathrm{CD} 11 \mathrm{c}^{+}$cells.

\section{Analysis of the Dendritic Cell Population in BAL Fluid}

BALB/c mice were sensitized with OVA, challenged by intranasal administration of OVA, and 2 months later given CIN treatment. They were again challenged with OVA and euthanized 18 hours later. Lungs were lavaged with $1 \mathrm{~mL}$ of PBS in two $0.5 \mathrm{~mL}$ aliquots introduced and withdrawn through the trachea. The BAL fluid was centrifuged 10 minutes at $300 \mathrm{~g}$, and cells were resuspended in PBS. Buffered paraformaldehyde was added to a final concentration of $4 \%$, and cells were fixed for 10 minutes at room temperature. Cells were washed with PBS and resuspended in PBS with 3\% FCS. Aliquots of the cell suspension were all stained for $\mathrm{CD} 11 \mathrm{c}$ and $\mathrm{CD} 11 \mathrm{~b}$ and individually for CD40, CD80, CD86, and I-Ad. Appropriate isotype controls and positive fluorescence markers for each fluor were also prepared. Cells expressing each of the four dendritic cell activation markers were counted by three-colour flow cytometry (FACSCalibur, $\mathrm{BD}$ Biosciences) gated on $\mathrm{CD} 11 \mathrm{c}^{+}$and $\mathrm{CD}_{11} \mathrm{~b}^{+}$cells.

\section{Analysis of the Dendritic Cell Population in Lymph Nodes}

$\mathrm{BALB} / \mathrm{c}$ mice were treated as described above for BAL fluid isolation, and peribronchial lymph nodes were removed at euthanasia. Single-cell suspensions were prepared by maceration of the lymph nodes through 40-micron cell strainers, and $\mathrm{CD} 11 \mathrm{c}^{+}$cells were isolated by magnetic bead separation. The cells were stained for CD11b and separately for each of the dendritic cell activation markers CD40, CD80, CD86, and I-Ad and were counted by flow cytometry (FACScan, BD Biosciences).

\section{IFN-Inducible Target Gene Array Analysis in Dendritic Cells}

To identify which genes were up- or downregulated by $\mathrm{CIN}$ treatment, CD11c ${ }^{+}$dendritic cells were isolated from lung cell suspensions of OVA-allergic/-challenged BALB/c mice (two mice per isolation) using anti-CD11c conjugated to magnetic beads (Miltenyi Biotech, Auburn, CA). Total ribonucleic acid (RNA) was purified from dendritic cells of mice treated with control vector or CIN, converted to cDNA using reverse transcriptase, and labelled with biotinylated deoxyuridine triphosphate (dUTP). The labelled cDNA was hybridized to a TranSignal Interferon-inducible Gene Array membrane (Panomics, Fremont, CA), and signals were detected by chemiluminescence on $\mathrm{x}$-ray film. The resulting film image was scanned, and densitometry calculations were done using the ScionImage (Scion Corporation, www.scioncorp. com) program to compare the results from untreated mice 
with those from CIN-treated mice. The gene array analysis was repeated once with a similar expression profile.

\section{Ribonuclease Protection Assay for Cytokine Gene Expression in Dendritic Cells}

$\mathrm{BALB} / \mathrm{c}$ mice were OVA-sensitized and challenged and given CIN treatment or chitosan-control vector only. After treatment, peribronchial lymph nodes were removed and macerated through 40-micron cell strainers. Single-cell suspensions were then mixed with anti-CD11c magnetic beads (Miltenyi Biotec, Auburn, CA), and bound cells were collected according to the manufacturer's protocol. Total RNA was prepared from the CD11c ${ }^{+}$cells, and ${ }^{32} \mathrm{P}-\mathrm{UTP}$ (Amersham, Piscataway, NJ)-labelled probes were generated by in vitro transcription of cytokine-specific multiprobe template sets (BD Pharmingen, San Diego, CA) using T7 RNA polymerase. The labelled probes were purified, adjusted to $3 \times 10^{5} \mathrm{cpm} / \mathrm{mL}$, and hybridized to $5 \mu \mathrm{g}$ of each RNA. The reactions were subsequently digested with ribonuclease (Rnase) followed by proteinase $\mathrm{K}$ and extracted with phenol-chloroform. After ethanol precipitation with 4 $\mathrm{M}$ ammonium acetate, the protected samples were resuspended in loading buffer and separated on a $6 \%$ Tris-borateEDTA (TBE)-urea gel (Novex Invitrogen, Carlsbad, CA). The gel was placed on filter paper, dried under a vacuum, and exposed to Kodak X-OMAT-AR film with intensifying screen at $-80^{\circ} \mathrm{C}$.

\section{Determination of Toll-Like Receptor mRNA Levels on Lymph Node Dendritic Cells}

A reverse transcriptase-polymerase chain reaction (RTPCR) was carried out on total RNA from $\mathrm{CD} 11 \mathrm{c}^{+}$cells isolated by magnetic bead separation from peribronchial lymph nodes of OVA-sensitized/OVA-challenged BALB/c mice treated with vector or CIN. PCR primers specific for the indicated Toll-like receptors (TLRs) were used along with $\beta$-actin as control, and amplification was performed for 25,30 , and 35 cycles.

\section{Statistical Analysis}

Each test group of mice contained at least four animals, and experiments were repeated twice. Values for all measurements are expressed as means \pm SEMs. Groups were compared by analysis of variance and through the use of paired Student $t$-tests. Differences between groups were considered significant at $p<.05$.

\section{Results and Discussion}

\section{Expression in the Lung of a Chitosan Nanoparticle- Delivered Plasmid}

To determine that pDNAs can be transported in chitosan nanoparticles and expressed in the lung epithelium, pEGFP was complexed with chitosan nanoparticles and administered intranasally to $\mathrm{BALB} / \mathrm{c}$ mice. At 1,3 , and 7 days thereafter, mice were euthanized, lungs were removed, and sections were examined by fluorescence microscopy for GFP expression. By 3 days after pEGFP nanoparticle administration, roughly half of the lung epithelial cells appeared to be positive for EGFP (data not shown), and expression of GFP continued for at least 7 days. We conclude that intranasal delivery of plasmids by means of chitosan nanoparticles results in a sustained expression of the encoded protein in the lung and provides an effective means of supplying therapeutic or prophylactic levels of an immunomodulatory molecule.

\section{Effects of CIN Therapy on T Lymphocytes}

In a previous study, we found that CIN treatment significantly lowered airway hyperresponsiveness to methacholine and reduced lung histopathology in a $\mathrm{BALB} / \mathrm{c}$ mouse model of OVA-allergic asthma. ${ }^{46} \mathrm{CIN}$ treated mice produced higher levels of IFN- $\gamma$ but less of the Th2 cytokines IL-4 and IL-5 and had reduced OVAspecific serum IgE compared with mice given vector alone. Lung infiltration by eosinophils was significantly reduced by CIN therapy, and overproduction of mucus was inhibited within 6 hours of CIN delivery by induced apoptosis of goblet cells. In this study, we sought to understand the mechanism of CIN action.

$\mathrm{T}$ cells play a critical role in the pathology of asthma and therefore may be potential targets of agents such as $\mathrm{CIN}$ for ameliorating asthmatic symptoms. $\mathrm{CD} 4^{+} \mathrm{T}$ helper cells can either promote (Th2) or inhibit (Th1) the inflammation of asthma depending on their phenotype and the presence or absence of specific cytokines. To examine the effects of CIN treatment on $\mathrm{CD}^{+}{ }^{+} \mathrm{T}$ lymphocyte populations in the lung, sections from control and CIN-treated mice were stained with rhodaminelabelled anti-T1/ST2L, specific for Th2 cells, or with FITC-labelled anti-IL-12R $\beta 2$ for Th1 cells. Sections were examined in a blinded fashion by fluorescence microscopy, and representative photographs were made to show comparisons of controls with CIN-treated animals. Lungs from CIN-treated mice showed qualitatively fewer Th2 
B.

A.

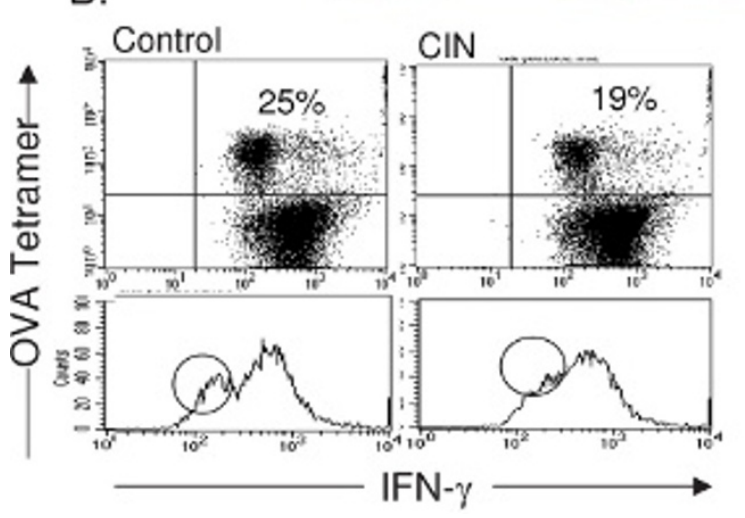

anti-T1/ST2L
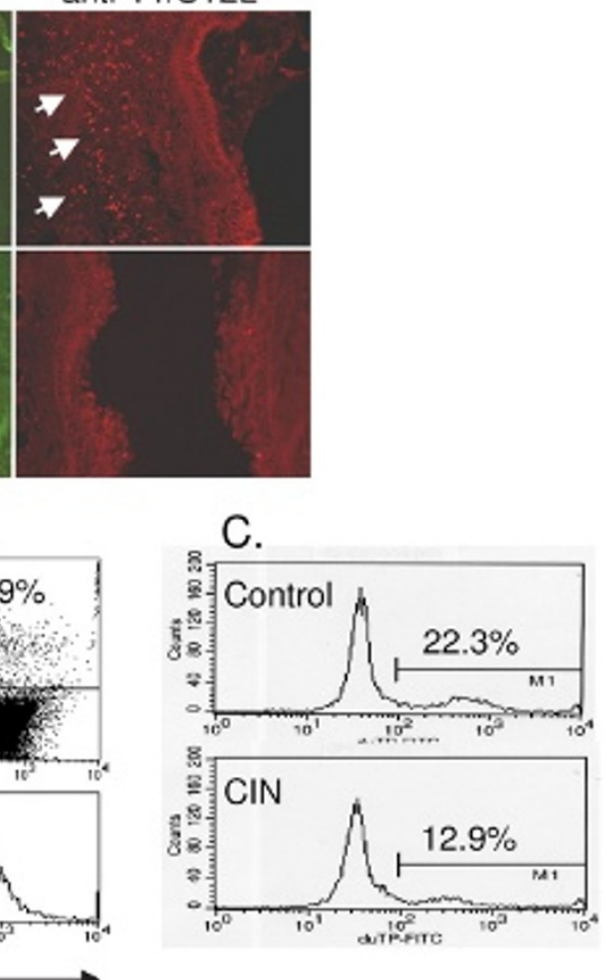

Figure 1. Chitosan interferon- $\gamma$ nanogene (CIN) modulates T-cell responses in ovalbumin (OVA)allergic mice. $A$, Localization of $\mathrm{T}$ helper cells in the lungs of C57BL/6 mice using antibody to interleukin12R $\beta$ (Th1) and T1/ST2L (Th2). B, Changes in interferon- $\gamma$ (IFN- $\gamma$ ) production by OVA-specific $\mathrm{CD}^{+} \mathrm{T}$ cells from the lung. T-cell receptor OVAspecific OT1 mice were treated with CIN and then sensitized with OVA and challenged 10 days later. $T$ lymphocytes were isolated from lungs, cultured with OVA, and stained with tetramer to label OVA-specific $\mathrm{CD}^{+}$ $\mathrm{T}$ cells. IFN- $\gamma$ was identified by intracellular cytokine staining and counted by flow cytometry after gating for tetramer-labelled cells. $C$, Apoptosis of OVA-specific $\mathrm{CD}^{+} \mathrm{T}$ cells from lungs determined by TUNEL assay and measured by flow cytometry. The data are based on cytometry of a minimum of 15,000 cells and were substantiated by a repeat experiment. cells and either the same or slightly more Th1 cells than control mice (Figure 1A).

Both $\mathrm{CD}^{+}$and $\mathrm{CD}^{+}{ }^{+} \mathrm{T}$ lymphocytes are involved in the immune response to allergens. To determine if CIN therapy affects the activity of antigen-specific $\mathrm{CD} 8^{+} \mathrm{T}$ cells, C57BL/6-OT1 mice carrying the transgene for the T-cell receptor specific for the OVA epitope, amino acids 257 to 264, were given intranasal CIN and then sensitized by intraperitoneal injection with OVA (day 1). They were again treated with CIN and then challenged with OVA intranasally on day 10. Lung lymphocytes were isolated 24 hours later and cultured in the presence of OVA and brefeldin A for 20 hours. Cell viability was $>80 \%$ (trypan blue dye exclusion test). The OVA-specific $\mathrm{CD} 8^{+} \mathrm{T}$ cells were stained using a tetramer for the 257- to 264-amino acid OVA peptide, and the IFN- $\gamma$ produced during OVA exposure was labelled by intracellular cytokine staining. After appropriate gating, the cells were counted by flow cytometry. The results (Figure 1B) show that CIN treatment caused a decrease in the number of IFN- $\gamma$ producing, OVA-specific $\mathrm{CD}^{+} \mathrm{T}$ cells in the lungs of OVA-challenged mice.

To further assess the fate of OVA-specific $\mathrm{CD}^{+} \mathrm{T}$ cells, OVA-allergic C57 mice were treated with CIN and 24 hours later challenged intranasally with OVA. Mice were euthanized 18 hours after challenge, and the lungs were used to prepare single-cell suspensions of $\mathrm{T}$ cells. The OVA-specific $\mathrm{CD}^{+}$T-cell population was labelled with phycoerythrin-tagged tetramer for OVA peptide conjugated with MHC-I. CD8 ${ }^{+}$cells were analyzed for apoptosis using the TUNEL assay (Promega) and counted by FACScan flow cytometry. The percentage of total OVAspecific $\mathrm{CD}^{+} \mathrm{T}$ cells that were apoptotic was lower in CIN-treated mice compared with untreated controls (Figure 1C); therefore, the decrease in IFN $-\gamma$ production observed in the $\mathrm{CD}^{+} \mathrm{T}$ cells does not appear to be the result of destruction of the cells but represents an IFN- $\gamma$ mediated downregulation of IFN- $\gamma$ production by a specific subpopulation of lymphocytes.

These CIN-mediated alterations in T-cell populations support the hypothesis that $\mathrm{CD}^{+} \mathrm{T}$ cells are important in allergen-induced lung pathology and that at least a part of the protective effect of CIN treatment can be attributed to a reduction in numbers of a specific $\mathrm{CD} 8^{+} \mathrm{T}$-cell population. Intranasal administration of antigen to rats was reported to induce T-cell activation concurrent with a burst of IFN- $\gamma$ production, whereas subsequent antigen exposure produced apoptosis and tolerization in a T-cell population. ${ }^{47}$ Also, IFN- $\gamma$ has been reported to induce apoptosis of $\mathrm{CD}^{+} \mathrm{T}$ cells. ${ }^{48,49}$ Our results in this OVA-allergic asthma model 
would suggest that IFN- $\gamma$ may have an autocrine effect, downregulating its own production in $\mathrm{T}$ lymphocytes.

\section{Effects of CIN Therapy on Dendritic Cells}

Antigen presentation is a key process in determining the magnitude of an immune response to an allergen such as OVA. Since antigen-presenting cells commonly take up extracellular particles, it is reasonable to suppose that CIN therapy, which uses chitosan nanoparticles, might involve cells that interact with or take up the particles and directly influence the immune response. Dendritic cells are the predominant antigen-presenting species in regulating $\mathrm{T}$ cell activation and thus may participate in the protective effect of CIN therapy.

To determine whether CIN therapy affects the activity of lung dendritic cells, CD11 $\mathrm{c}^{+}$mononuclear cells were isolated from lungs of OVA-allergic mice with or without CIN treatment and incubated with control nanoparticles alone or with CIN. Flow cytometry was done on cell suspensions after labelling for CD11c and for each of the
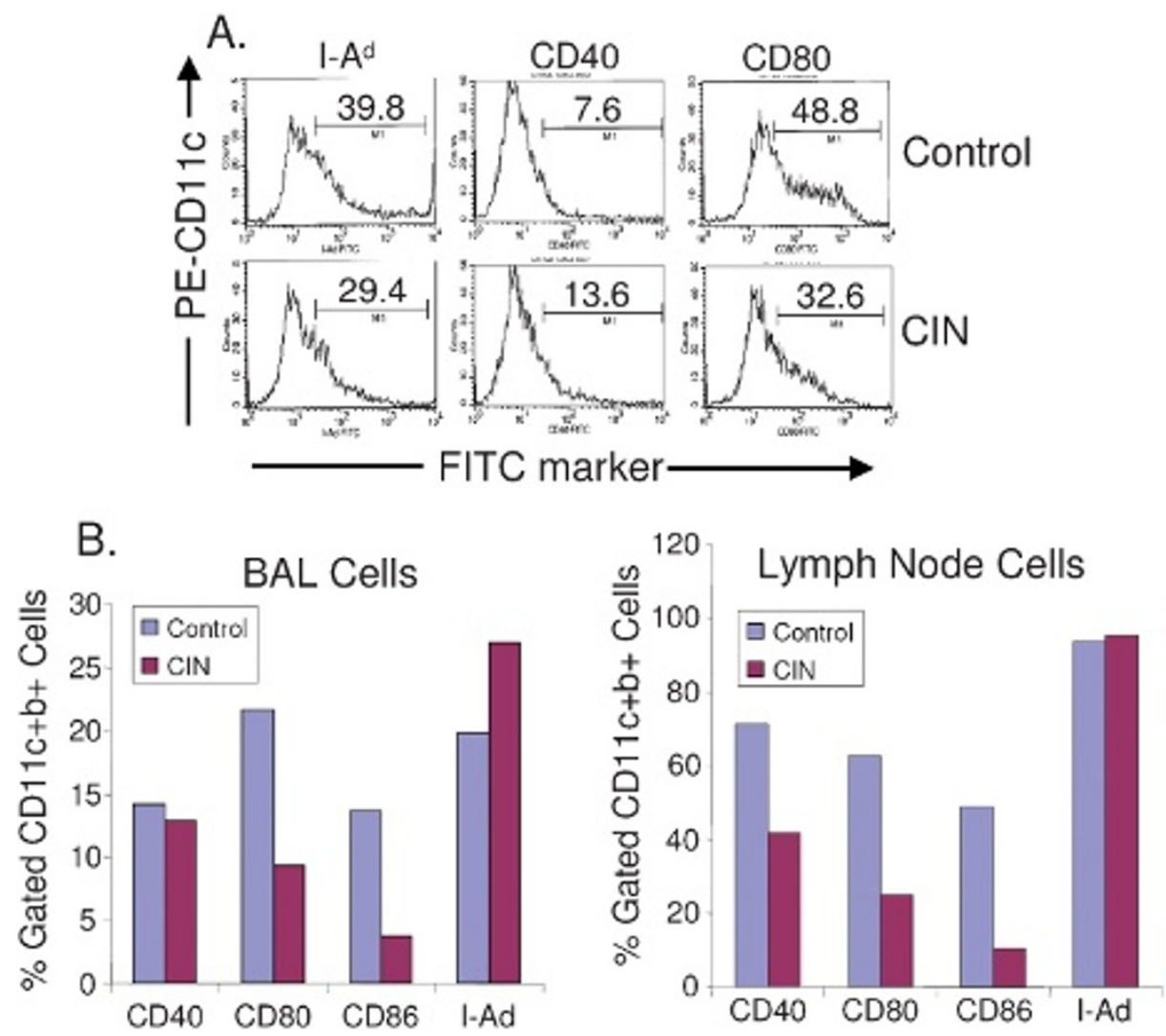

following markers: I-Ad, CD40, and CD80. The results showed an increase in CD40 but a decrease in expression of CD80 and I-Ad in dendritic cells from CIN-treated mice compared with controls (Figure 2A). CD40 is a cell surface receptor related to the tumour necrosis factor receptor superfamily that binds to CD40 ligand, which is expressed primarily by $\mathrm{T}$ cells. Upregulation of CD40 may influence the interaction of lung dendritic cells with allergen-specific T lymphocytes.

To further examine the potential effects of CIN on dendritic cells, ex vivo cultures from BAL fluid and from thoracic lymph nodes were prepared from mice with or without CIN treatment and tested for expression of CD40, the costimulatory molecules CD80 and CD86, and I-Ad (MHC-II). The results showed that CIN treatment decreased expression of CD80 and CD86 on dendritic cells from both BAL fluid and lymph nodes (Figure 2B). CD40 expression was decreased in lymph node dendritic cells, whereas the expression of I-Ad was unaffected in both DC types. These results indicated that dendritic cells from lung lavage or from peribronchial lymph nodes can

Figure 2. Effect of chitosan interferon- $\gamma$ nanogene (CIN) therapy on expression of dendritic cell activation markers. $A$, In vitro CIN treatment of CD11c ${ }^{+}$cells from the lungs of ovalbumin (OVA)-allergic mice. $\mathrm{BALB} / \mathrm{c}$ mice were OVA sensitized and challenged with OVA and sacrificed 18 hours later. Lungs were removed and $\mathrm{CD} 11 \mathrm{c}^{+}$dendritic cells were isolated as described in Materials and Methods. Dendritic cells were cultured with vector alone (control) or with CIN. Flow cytometry was performed on cell suspensions after labelling for CD11c (fluorescein isothiocyanate [FITC]) and for each of the following markers: I-Ad, CD40, and CD80 phycoerythrin (PE). Counts were done using a FACScan gated for CD11c cells. The figure shows the percentage of cells that were positive for CD11c and for each of the markers. Activation marker expression in $\mathrm{CD} 11 \mathrm{c}^{+}$cells from the bronchoalveolar lavage (BAL) fluid and lymph nodes of OVA-allergic mice treated with CIN (B). Mice were treated as described in Materials and Methods, and $\mathrm{CD} 11 \mathrm{c}^{+}$cells were isolated from BAL fluid and lymph nodes. Cells were analyzed by flow cytometry for the expression of CD40, CD80, CD86, and I-Ad gated to CD11 $\mathrm{c}^{+} \mathrm{b}^{+}$. The data are based on cytometry of a minimum of 15,000 cells and were substantiated by a repeat experiment. 
be partially inactivated by CIN treatment, and their capacity to activate $\mathrm{T}$ cells may consequently be reduced.

Survival of mature dendritic cells is necessary for subsequent interaction with $\mathrm{T}$ lymphocytes, and CIN may affect apoptosis of dendritic cells and their activation. To examine this possibility, OVA-allergic mice were challenged with OVA and then 2 months later given CIN and challenged again with OVA. Mononuclear cells were isolated from lungs as described above, and the relative numbers of $\mathrm{CD} 11 \mathrm{c}^{+}$and $\mathrm{CD} 11 \mathrm{~b}^{+}$cells were determined by flow cytometry. CIN treatment decreased the number of CD11 $c^{+} b^{+}$cells and increased the number of CD11 $c^{+} b^{-}$ cells (Figure 3). CD11 $c^{+} b^{+}$cells are considered to be the most active antigen-presenting cells, so the observation that CIN therapy caused a significant reduction (fivefold) in the numbers of CD11 $c^{+} b^{+}$cells is consistent with the other data in supporting the idea that CIN treatment decreases the inflammatory response to an allergen by inhibiting dendritic cell activation of OVA-specific $\mathrm{T}$ cells.

\section{Alteration of Dendritic Cell Gene Expression by CIN Treatment}

Although CD40, CD80, and CD86 are key markers of activated dendritic cells, many other genes are important in the allergic immune response and may be up- or downregulated by CIN treatment. To determine how CIN affects gene expression, total RNA was isolated from the lung dendritic cells of control and CIN-treated mice, converted to cDNA, and labelled as probes, which were hybridized to a mouse TranSignal Interferon-inducible Gene Array membrane. The x-ray film images of the control and CIN arrays were scanned, and spot densities were analyzed and compared using the ScionImage program. A two- to threefold increase or decrease in spot density was considered significant, and an example of some CIN-upregulated genes is shown in Figure 4A. The results demonstrate that such arrays can be useful in detecting significant changes in gene expression and that novel changes can be identified in lung dendritic cells from CIN-treated mice compared with controls. To confirm CIN-mediated changes in expression of selected cytokine genes, the RNAs were analyzed further by RNase protection assay. The results show that CIN treatment augments expression of IL-12 p40, IL-18, IL- $1 \alpha$, and IFN- $\gamma$ in dendritic cells (Figure 4B). Dendritic cells are activated by signals generated through recognition of foreign antigens by their surface TLRs. To examine the possibility that the observed changes in gene expression were due to CINmediated differential expression of TLR genes, the expression of a number of TLR genes on peribronchial lymph node dendritic cells (purified by CD11c magnetic beads) was assayed by RT-PCR. The results indicated that CIN treatment did not affect expression of TLR-2, -4, -5, -6 , and -9 at the mRNA level (Figure 5) and, therefore, that CIN affects the activation of dendritic cells independently of TLR signalling.

\section{Conclusion}

These studies demonstrate that IFN- $\gamma$ delivered via intranasal CIN treatment reduces the allergic immune response by means of its effects on $\mathrm{CD}^{+} \mathrm{T}$ cells and dendritic cells in mice. The results are consistent with a mechanism whereby CIN therapy decreases the innate immune response by altering cytokine production of a $\mathrm{CD}^{+} \mathrm{T}$-cell subpopulation and by decreasing the antigenpresenting activity of dendritic cells.

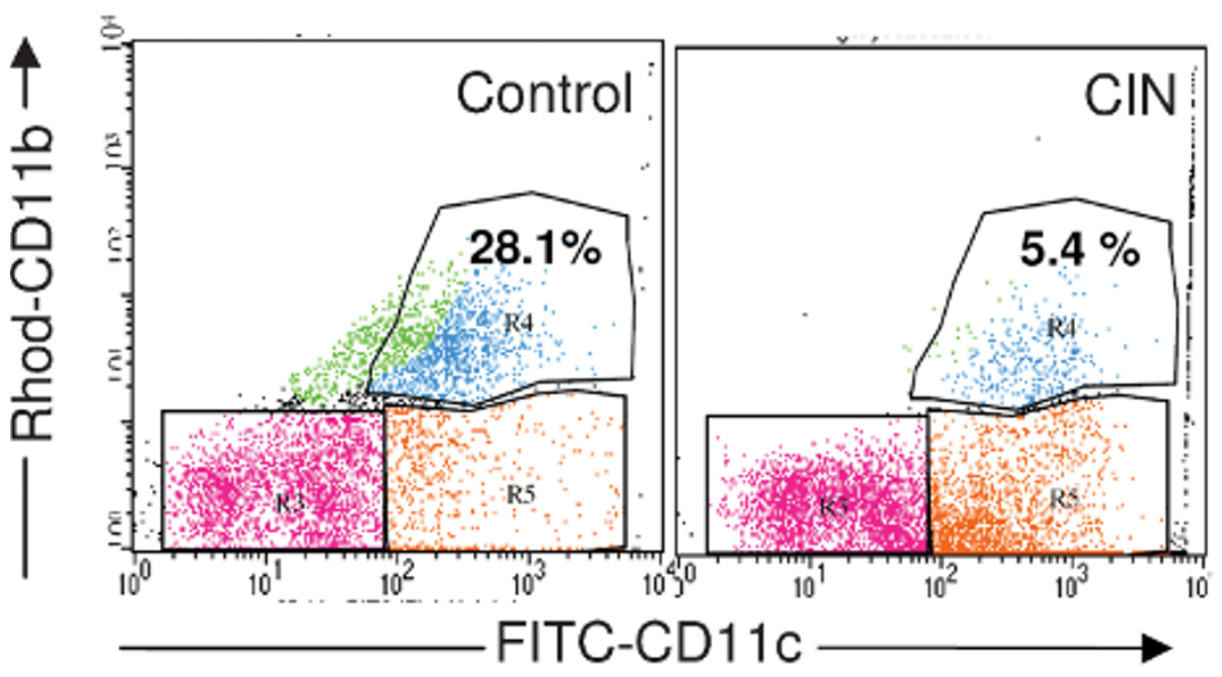

Figure 3. Effect of chitosan interferon$\gamma$ nanogene (CIN) therapy on the dendritic cell population in the lung. Ovalbumin-allergic/-challenged mice were treated with or without CIN therapy as described in Materials and Methods, and mononuclear cells were isolated from the lungs. Cells were stained with fluorescein isothiocyanate (FITC)-anti-CD11c and rhodamine (Rhod)-anti-CD11b, and CD11 $c^{+} b^{+}$ cells were counted by flow cytometry. Values given are the percentage of total

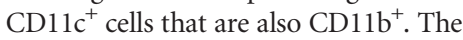
data are based on cytometry of a minimum of 15,000 cells and were substantiated by a repeat experiment. 


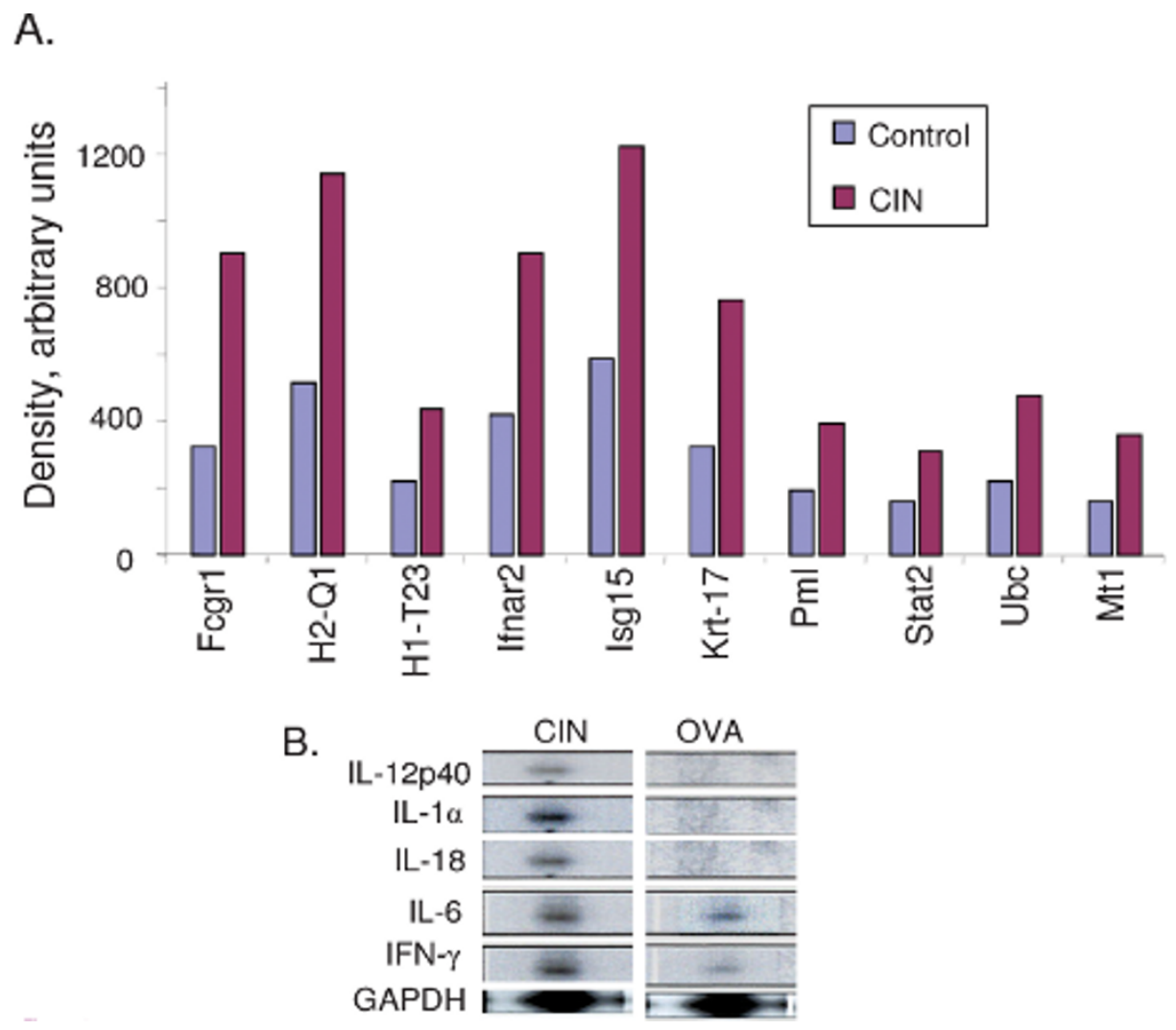

Figure 4. Chitosan interferon- $\gamma$ nanogene $(\mathrm{CIN})$ treatment induces changes in gene expression in lung dendritic cells. $A$, Total ribonucleic acid (RNA) from dendritic cells of ovalbumin (OVA)-allergic/-challenged mice with or without CIN treatment was isolated and assayed with the mouse TranSignal Interferon-inducible Gene Array Kit, as described in Materials and Methods. The arrays were scanned and the images analyzed using the ScionImage densitometry program. A two- to threefold increase or decrease in spot density was considered significant, and a selection of genes upregulated by CIN treatment is shown. B, Ribonuclease protection assay for CIN-induced cytokine gene expression in dendritic cells. Total RNA was isolated from $\mathrm{CD} 11 \mathrm{c}^{+}$lymph node dendritic cells of OVA-allergic/-challenged control and CIN-treated mice and hybridized to probes specific for interleukin (IL)-12 (p40), IL-1 $\alpha$, IL-18, IL-6, and interferon (IFN)- $\gamma$. The housekeeping gene, GAPDH, was included as a normalization control. The data presented are from one typical experiment of two performed. Fcgr1 = a high-affinity receptor for immunoglobulin G; H1-T23 = histocompatibility t23 region; H2-Q1 = histocompatibility 2-Q region; Ifnar2 = interferon- $\alpha$ receptor 2; Isg15 = interferon-induced protein, 15 $\mathrm{kDa}$; Krt-17 = keratin-17; Mt1 = metallothionein 1; Pml = promyelocytic leukemia gene; Stat $2=$ signal transducer and activator of transcription 2; $\mathrm{Ubc}=$ ubiquitin $\mathrm{C}$.

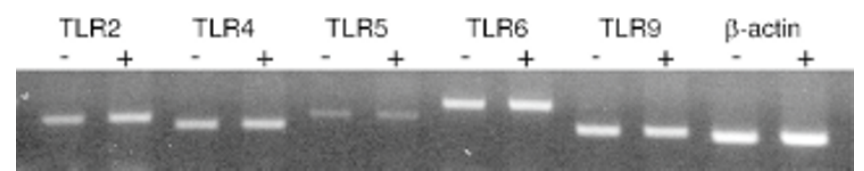

Figure 5. Expression of Toll-like receptors (TLRs) on dendritic cells. Total ribonucleic acid (RNA) was purified from CD11c ${ }^{+}$cells isolated by magnetic bead separation from peribronchial lymph nodes of mice with or without chitosan interferon- $\gamma$ nanogene treatment. RNAs were reverse-transcribed and used as a template for reverse transcriptasepolymerase chain reaction (PCR) with primer pairs and PCR conditions specific for the indicated TLRs. The results of PCRs for 25 cycles are shown with $\beta$-actin used as a control (one typical experiment of two).

\section{Acknowledgement}

We would like to thank Sylvia Montalvo for her assistance in preparation of the manuscript.

\section{References}

1. Behera AK, Kumar M, Lockey RF, Mohapatra SS. Adenovirusmediated interferon gamma gene therapy for allergic asthma: involvement of interleukin 12 and STAT4 signaling. Hum Gene Ther 2002;13:1697-709.

2. Monahan PE, Samulski RJ. AAV vectors: is clinical success on the horizon? Gene Ther 2000;7:24-30.

3. Senior K. Adeno-associated virus vectors under scrutiny. Lancet 2002;359(9313):1216.

4. Zaiss AK, Liu Q, Bowen GP, et al. Differential activation of innate immune responses by adenovirus and adeno-associated virus vectors. J Virol 2002;76:4580-90.

5. Zhao N, Liu DP, Liang CC. Hot topics in adeno-associated virus as a gene transfer vector. Mol Biotechnol 2001;19:229-37.

6. Cohen AD, Boyer JD, Weiner DB. Modulating the immune response to genetic immunization. FASEB J 1998;12:1611-26.

7. Donnelly JJ, Ulmer JB, Liu MA. DNA vaccines. Dev Biol Stand 1998;95:43-53. 
8. Erbacher P, Zou S, Bettinger T, et al. Chitosan-based vector/DNA complexes for gene delivery: biophysical characteristics and transfection ability. Pharm Res 1998;15:1332-9.

9. Lee KY, Kwon IC, Kim YH, et al. Preparation of chitosan selfaggregates as a gene delivery system. J Control Release 1998;51: 213-20.

10. Leong KW, Mao HQ, Truong-Le VL, et al. DNA-polycation nanospheres as non-viral gene delivery vehicles. J Control Release 1998;53:183-93.

11. Richardson SC, Kolbe HV, Duncan R. Potential of low molecular mass chitosan as a DNA delivery system: biocompatibility, body distribution and ability to complex and protect DNA. Int J Pharm 1999;178:231-43.

12. Roy K, Mao HQ, Huang SK, Leong KW. Oral gene delivery with chitosan-DNA nanoparticles generate immunologic protection in a murine model of peanut allergy. Nat Med 1999;5:387-91.

13. Nishimura K, Nishimura S, Nishi N, et al. Immunological activity of chitin and its derivatives. Vaccine 1984;2:93-9.

14. Otterlei M, Varum KM, Ryan L, Espevik T. Characterization of binding and TNF-alpha-inducing ability of chitosans on monocytes: the involvement of CD14. Vaccine 1994;12:825-32.

15. Muzzarelli R, Baldassarre V, Conti F, et al. Biological activity of chitosan: ultrastructural study. Biomaterials 1988;9:247-52.

16. Pappineau A, Hoover D, Knoor D, Farkas D. Food biotechnol. New York (NY); Marcel Dekker; 1991;5:45-7.

17. Aspden TJ, Mason JD, Jones NS, et al. Chitosan as a nasal delivery system: the effect of chitosan solutions on in vitro and in vivo mucociliary transport rates in human turbinates and volunteers. J Pharm Sci 1997;86:509-13.

18. Bodmeier R, Chen HG, Paeratakul O. A novel approach to the oral delivery of micro- or nanoparticles. Pharm Res 1989;6:413-7.

19. Illum L, Farraj NF, Davis SS. Chitosan as a novel nasal delivery system for peptide drugs. Pharm Res 1994;11:1186-9.

20. Miyazaki S, Nakayama A, Oda M, et al. Chitosan and sodium alginate based bioadhesive tablets for intraoral drug delivery. Biol Pharm Bull 1994;17:745-7.

21. Tozaki H, Komoike J, Tada C, et al. Chitosan capsules for colonspecific drug delivery: improvement of insulin absorption from the rat colon. J Pharm Sci 1997;86:1016-21.

22. Artursson P, Lindmark T, Davis SS, Illum L. Effect of chitosan on the permeability of monolayers of intestinal epithelial cells (Caco2). Pharm Res 1994;11:1358-61.

23. Coyle AJ, Tsuyuki S, Bertrand C, et al. Mice lacking the IFNgamma receptor have impaired ability to resolve a lung eosinophilic inflammatory response associated with a prolonged capacity of T cells to exhibit a Th2 cytokine profile. J Immunol 1996;156:2680-5.

24. Romagnani S. Lymphokine production by human T cells in disease states. Annu Rev Immunol 1994;12:227-57.

25. Wenner CA, Guler ML, Macatonia SE, et al. Roles of IFN-gamma and IFN-alpha in IL-12-induced T helper cell-1 development. J Immunol 1996;156:1442-7.

26. Szeto C, Gillespie KM, Mathieson PW. Levamisole induces interleukin-18 and shifts type 1/type 2 cytokine balance. Immunology 2000;100:217-24.

27. Gao Y, Chenping Z, Lin XP. [Aerosolized recombinant interferongamma prevent antigen-induced eosinophil recruitment in guinea pig trachea]. Zhonghua Jie He He Hu Xi Za Zhi 1997;20:287-90.
28. Boraschi D, Censini S, Bartalini M, et al. Interferons inhibit LTC4 production in murine macrophages. J Immunol 1987;138:4341-6.

29. Chen H, Munakata M, Amishima M, et al. Gamma-interferon modifies guinea pig airway functions in vitro. Eur Respir J 1994;7: 74-80.

30. van der Pouw Kraan TC, Boeije LC, de Groot ER, et al. Reduced production of IL-12 and IL-12-dependent IFN-gamma release in patients with allergic asthma. J Immunol 1997;158:5560-5.

31. Daines MO, Hershey GK. A novel mechanism by which interferongamma can regulate interleukin (IL)-13 responses. Evidence for intracellular stores of IL-13 receptor alpha -2 and their rapid mobilization by interferon-gamma. J Biol Chem 2002;277:1038793.

32. Ford JG, Rennick D, Donaldson DD, et al. IL-13 and IFN-gamma: interactions in lung inflammation. J Immunol 2001;167:176977.

33. Krasnowska M, Medrala W, Malolepszy J, Krasnowski R. Effect of recombinant IFN-gamma on IgE-dependent leukotriene generation by peripheral blood leukocytes in patients with pollinosis and asthma. Arch Immunol Ther Exp (Warsz) 2000;48:287-92.

34. Pierkes M, Bellinghausen I, Hultsch T, et al. Decreased release of histamine and sulfidoleukotrienes by human peripheral blood leukocytes after wasp venom immunotherapy is partially due to induction of IL-10 and IFN-gamma production of T cells. J Allergy Clin Immunol 1999;103(2 Pt 1):326-32.

35. Gurujeyalakshmi G, Giri SN. Molecular mechanisms of antifibrotic effect of interferon gamma in bleomycin-mouse model of lung fibrosis: downregulation of TGF-beta and procollagen I and III gene expression. Exp Lung Res 1995;21:791-808.

36. Cohen J. IL-12 deaths: explanation and a puzzle. Science 1995;270: 908.

37. Mohapatra SS. IL-12 possibilities. Science 1995;269:1499.

38. Hogan SP, Foster PS, Tan X, Ramsay AJ. Mucosal IL-12 gene delivery inhibits allergic airways disease and restores local antiviral immunity. Eur J Immunol 1998;28:413-23.

39. Murray HW. Current and future clinical applications of interferon-gamma in host antimicrobial defense. Intensive Care Med 1996;22 Suppl 4:S456-61.

40. Sur S, Lam J, Bouchard P, et al. Immunomodulatory effects of IL12 on allergic lung inflammation depend on timing of doses. J Immunol 1996;157:4173-80.

41. Dow SW, Schwarze J, Heath TD, et al. Systemic and local interferon gamma gene delivery to the lungs for treatment of allergen-induced airway hyperresponsiveness in mice. Hum Gene Ther 1999;10:1905-14.

42. Li XM, Chopra RK, Chou TY, et al. Mucosal IFN-gamma gene transfer inhibits pulmonary allergic responses in mice. J Immunol 1996;157:3216-9.

43. Okubo T, Hagiwara E, Ohno S, et al. Administration of an IL-12encoding DNA plasmid prevents the development of chronic graftversus-host disease (GVHD). J Immunol 1999;162:4013-7.

44. Tovey MG, Maury C. Oromucosal interferon therapy: marked antiviral and antitumor activity. J Interferon Cytokine Res 1999;19: 145-55.

45. Kumar M, Behera AK, Lockey RF, et al. Intranasal gene transfer by chitosan-DNA nanospheres protects BALB/c mice against acute respiratory syncytial virus infection. Hum Gene Ther 2002;13: $1415-25$. 
46. Kumar M, Kong X, Behera AK, et al. Chitosan IFN-gamma-pDNA nanoparticle (CIN) therapy for allergic asthma. Genet Vaccines Ther 2003;1:3.

47. Laliotou B, Duncan L, Dick AD. Intranasal administration of retinal antigens induces transient $\mathrm{T}$ cell activation and apoptosis within drainage lymph nodes but not spleen. J Autoimmun 1999;12:145-55.
48. Badovinac VP, Tvinnereim AR, Harty JT. Regulation of antigenspecific CD8+ $\mathrm{T}$ cell homeostasis by perforin and interferongamma. Science 2000;290:1354-58.

49. Lohman BL, Welsh RM. Apoptotic regulation of $\mathrm{T}$ cells and absence of immune deficiency in virus-infected gamma interferon receptor knockout mice. J Virol 1998;72:7815-21. 